\title{
Performance Analysis of Two Dimensional Wavelength/ Time Encoding System for Optical CDMA Networks
}

\author{
Awab Fakih, Member, IACSIT, Shraddha Panbude, and Santosh Jagtap
}

\begin{abstract}
Optical code-division multiple access (OCDMA) combines the large bandwidth of the fiber medium with the flexibility of the CDMA technique to achieve the high-speed connectivity. OCDMA has the potential to support asynchronous, bursty communications. The establishment of OCDMA needs to overcome the cardinality problem of 1-D codes. In order to increase the number of users, unipolar codes with larger capacity need to be designed. In this paper we have analyzed the performance of an incoherent OCDMA scheme based on wavelength/time codes. This paper shows that 2-D codes have better spectrum efficiency than 1-D OCDMA codes of the same cardinality.
\end{abstract}

Index Terms-Code-division multiple access (CDMA), unipolar codes, wavelength hopping/time spreading (W/T), encoding, cardinality, multiple access interference (MAI).

\section{INTRODUCTION}

The TDMA, WDMA and CDMA are available major multiple access techniques. Both TDMA and WDMA system require time or frequency management system however the CDMA system does not require any centralized control and thereby reduce the complexity of the system. Code division multiple access (CDMA) technique enables several users to have access to the network simultaneously and asynchronously by allocating a specific code to each user. CDMA systems offer simplified network control and high bandwidth efficiency at a low costs. Successful achievements and widespread applications of the CDMA in the wireless area have renewed interest in investigating its application in the optics communication systems. Optical CDMA (OCDMA) has for a long time been the subject of research due to its inherent ability to support asynchronous burst communications. Initially OCDMA was employed for local area [1], then for access network applications [2], [3] and currently for emerging optical networks such as generalized multiprotocol label switching, fiber-to-home (FTTH) or fiber-to-the-curb (FTTC) [4]-[6].

In particular, when a large number of simultaneous network subscribers increases, the MAI increases, resulting in system performance degradation. As a solution to this OCDMA impediment, the number of chips collision in the receiver must be reduced. In practice, the length of the code should be increased and the weight of the code (chips that have value 1)

Manuscript received January 11, 2014; revised May 12, 2014.

Awab Fakih is with the Electronics and Telecommunication Department, Anjuman-I-Islamic Kalsekar Technical Campus, Panvel, India (email: awaabfakih0@gmail.com).

Shraddha Panbude and Santosh Jagtap are with the Electronics and Telecommunication Department, Vidyalankar Institute of Technology, Wadala, India. should be decreased. Consequently, the increase of length of one dimensional unipolar code generates further limitations where complexity of the encoder and decoder implementation increases. As a result of this, the data rate transmission decreases. To avoid such system limitations, we use 2-D unipolar codes [7]. In general, 2-D unipolar codes can be constructed by combining 1-D code and temporal spreading and hopping frequency or by using a spatial/frequencies schema. In general two coding techniques are combined to create 2-D codes. In this paper we combine spectrum encoding with temporal encoding to create wavelength hopping/ time spreading (W/T) 2-D codes.

This paper is organized as follows; in Section II a brief description of the state of the art of the OCDMA technique is presented. In Section III, a study of the optical codes' features and their characteristics is reported. On the other hand, Section IV is devoted to analyzing the system's performance of 2-D codes deployed in OCDMA systems. Finally, conclusions are drawn in Section V.

\section{OpticAl Code Division MultiPle AcCEss (OCDMA)}

Optical Code Division Multiple Access (OCDMA) is technology to realize multiplexing transmission and multiple accesses by coding in optical domain, which supports multiple simultaneous transmissions in the same timeslot and same frequency. It is another technology of multiplexing and multiple accesses besides Optical Time Division Multiplexing (OTDM) and Wavelength Division Multiplexing (WDM) and a potentially promising technique for optical networks in the future, and especially, due to its easy access and flexible network structure, it is very applicable to the access network.

OCDMA has become a promising technology to implement truly all optical communication and networking that uses optical signal processing directly, combining the advantage of electrical CDMA with the bandwidth predominance of fiber-optic and optical signal processing devices. The Passive optical access network, LAN and WAN, can be built up by using OCDMA technology The combination of OCDMA with WDM or TDM can enhance signal multiplexing and label switching through the combination of OCDMA with WDM or/and IP over WAN so that transmission and switching capacity of network can be enhanced and performance of the communication network can be heightened.

OCDMA can implement high-speed transmission, switching and add/drop of data through using all-optical signal processing and thus it can realize all-optical communication and all-optical networking and overcome the 
effect of electronic bottleneck, which exists in the electronic node in the traditional network. OCDMA has the following advantages:

- Subscribers can access the network at random and the network has soft capacity.

- OCDMA does not need buffering in queue because it uses the tell-to-go protocol.

- OCDMA network can assign the bandwidth and used optical network bandwidth effectively.

- The traffic protocol and network topology are transparent in the OCDMA network.

- An OCDMA network is somewhat secure and cryptic for the transmission information.

- OCDMA is highly compatible with DWDM and TDM.

OCDMA networks employ distributed management, which is simple and it is convenient to locate network failure and protect and recover. Because of the advantages mentioned above, OCDMA can support multimedia including voice, data, video, including IP traffic, video-on-demand, streaming media.

Optical CDMA systems can be divided into two broad categories based on the way in which a particular user's code is applied to the optical signal. In a coherent OCDMA system, a given user's code is generally applied via phase coding of the optical signal field, which is often derived from a highly coherent wideband source, such as a mode-locked laser. The receiver for a coherent OCDMA system relies on a coherent reconstruction of the signal field to recover the decoded user's data. In this the signals are bipolar in nature. Coherent systems achieve higher performance than the incoherent ones but require high-precision control of the optical path within the encoder and decoder In contrast an incoherent OCDMA system typically relies on amplitude-modulated codes rather than directly manipulating the optical phase. Also, the receiver is based upon an incoherent decoding and recovery process. A number of incoherent OCDMA system architectures utilize wideband incoherent sources, such as a broadband amplified spontaneous emission source, while other incoherent architectures utilize coherent laser sources as part of their implementation. Incoherent systems are regarded as more practical because the light sources and encoding and decoding techniques have low complexity and are cost effective.

For optical encoding and decoding in incoherent OCDMA unipolar codes are used. Whereas, bipolar codes that are used in RF CDMA (Radio Frequency Code Division Multiple Access) have poor performance in OCDMA system and cannot be used. Therefore, unipolar codes with good system performance need to be developed. Consequently, in the earlier years, the research on incoherent OCDMA focused on looking for and obtaining unipolar codes with good auto-and cross-correlations, such as OOC (Optical Orthogonal/Pseudo-Orthogonal Codes) [1], [8], PC (Prime Codes), QCC (Quadratic Congruence Codes) [9] etc. In order to improve the performances of PC and QCC, EPC (Extended Prime Codes) [10], MPC (Modified Prime Codes) [11] and EQCC (Extended Quadratic Congruence Codes) [12] have been proposed again. Among these one dimensional codes, OOC has the best performance but its construction is complicated, compared with those of other codes [13].

\section{WAVELENGTH HOPPING TIME SPREADING (WH/TS) SYSTEM}

Up till now, various 1-D codes for the OCDMA systems are proposed and compared theoretically but comparison of codes on the basis of data modulation format have been rarely done. The existing 1-D codes are having restrictions on code lengths and weights. Two dimensional codes operate in both time and wavelength domain can be a good solution to the limitations offered by one dimensional codes. Various approaches have been suggested for design of 2-D codes. The wavelength/time codes are here constructed using folded optimum Golomb ruler sequences [14], [15].

Wavelength-Hopping Time-Spreading (WHTS) system is a 2-D coding approach that spreads the codes in both the time and wavelength domains simultaneously. The general schematic of an OCDMA network is shown in the transmitter are the source and the encoder while the receiver consists of the decoder and the receiver electronics. In 2-D OCDMA, pulses are placed in different chips across the bit period and each chip is of different wavelength, thus following a wavelength- hopping pattern, achieving increased code design flexibility as well as code performance. Thus, WHTS codes can be represented as code matrices with time and wavelength as its two axes. In spite of the use of orthogonal codes, the main effect limiting the effective signal-to-noise ratio of the overall system is the interference resulting from the other users transmitting at the same time, which is called Multiple Access Interference (MAI). MAI is the major source of noise in OCDMA systems [16]. The number of users in a WHTS OCDMA network has a soft limit with a graceful degradation of performance with increasing number of users [17]. (See Fig. 1).

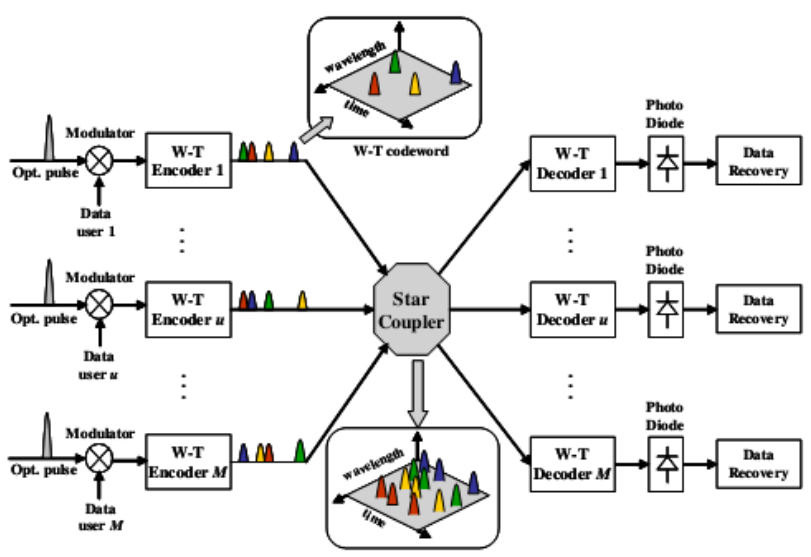

Fig. 1. Typical diagram of wavelength/ time OCDMA network.

\section{PERFORMANCE OF TWO DIMENSIONAL W/T CODES IN OCDMA SYSTEM}

In this paper we have designed an encoder for W/T OCDMA system. This can be used for up to 16 users. The block diagram of the encoder is shown in Fig. 2. Here we have demonstrated an incoherent OCDMA system based on a wavelength-time spreading coding technique. The $2-\mathrm{D}$ W/T 
code has been redesigned by using four wavelength and ten time slot in the system. Four mode locked laser have been used to create a WDM multi-frequency light source. This carrier signal is used to modulate the PRBS data of the user. After modulation an encoder is used for encoding the signal. Mode locked laser is used for generating pulses of pulse width of $\mathrm{e}$ at repetition rate equal to data rate of the system. The wavelength is range from $1550 \mathrm{~nm}$ to $1552 \mathrm{~nm}$, with $0.4 \mathrm{~nm}$ wavelength spacing.

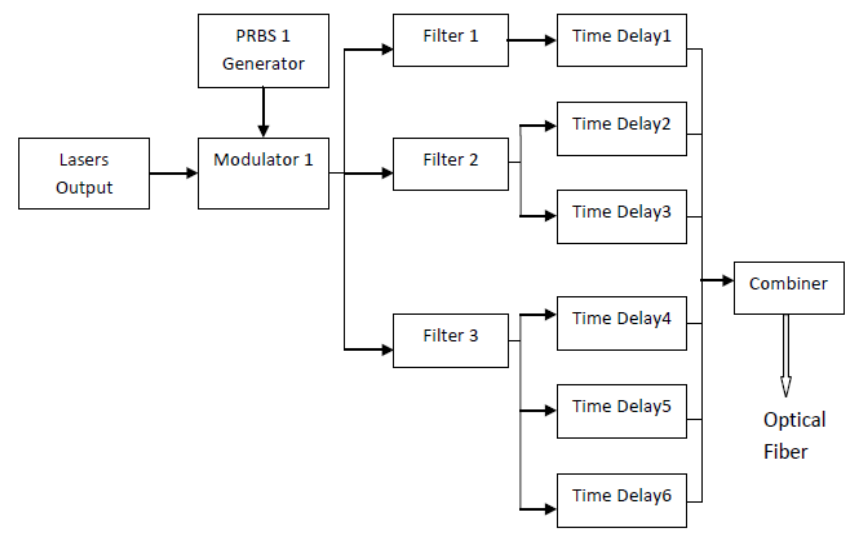

Fig. 2. Encoder design of optical CDMA system.

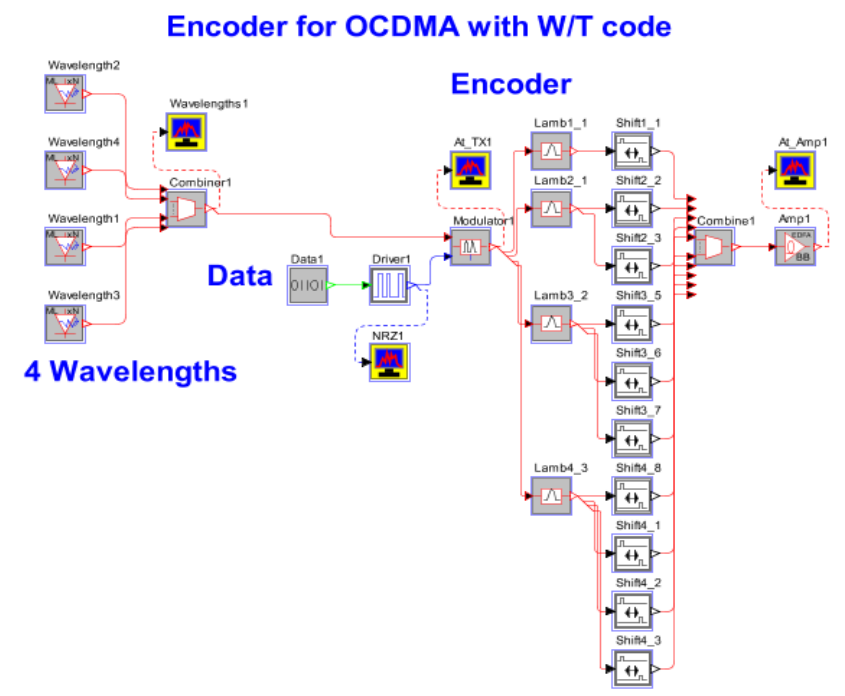

Fig. 3. Encoder for OCDMA with W/T code.

The PRBS data generator is used to generate random data of 26-pattern length. An electrical NRZ signal generator is used to convert digital data into electrical signal. A Mach-Zehnder LiNbO3 modulator modulates the multiplexed four wavelengths according to the NRZ electrical data.

The modulated signal is given to the encoder. In an encoder four optical filters and ten shift signals are used to produce the encoded bit stream. The optical filter is used to filter out one spectral wavelength and then the shifting signal is used to produce a pulse at specified chip. The encoder used delay line arrays providing delays in terms of integer multiples of chip times. The combiner combines ten of the displaced pulses to from an encoded signal. The diagram of encoder is shown in Fig. 3.

The input data to the encoder is shown in Fig. 4. As shown in the diagram the input data is NRZ line coded. In this paper we have analyzed the performance of $\mathrm{W} / \mathrm{T}$ based encoder with
PSO based encoder. The encoder for OCDMA with PSO code is as shown in Fig. 5. The signal magnitude of the output of PSO based system is $50 \mathrm{~mW}$ and that of W/T based system is approximately $40 \mathrm{~mW}$ as shown in Fig. 6 and Fig. 7 respectively.

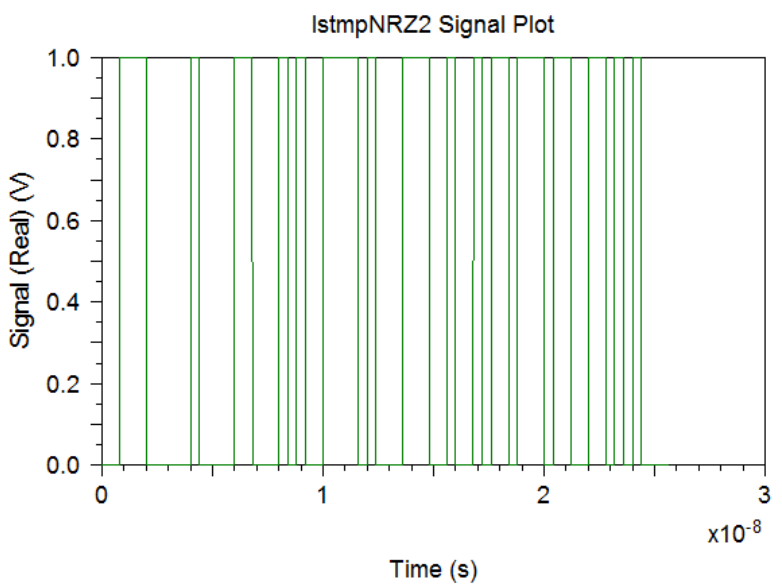

Fig. 4. NRZ coded input data.

\section{Encoder for OCDMA with PSO code}

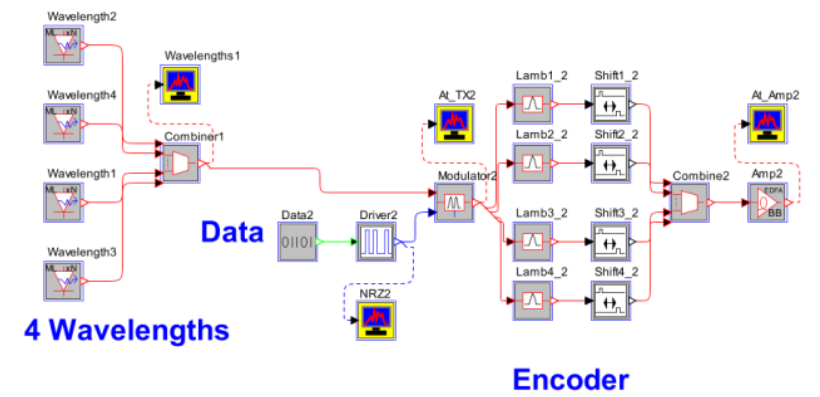

Fig. 5. Encoder for OCDMA with PSO code.

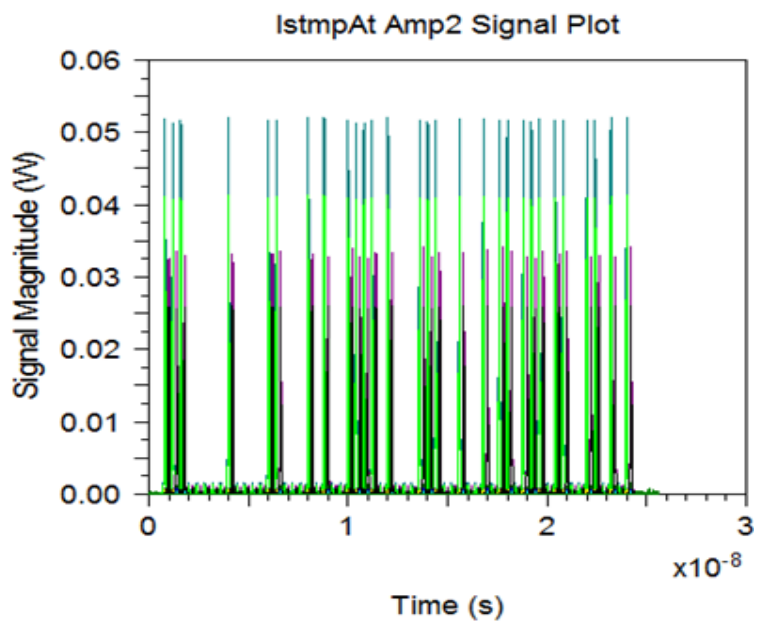

Fig. 6. Encoder output with PSO code.

However this can be increased by the use of an optical amplifier, either an EDFA or Raman Amplifier. The wavelength spectrum of both the systems is shown in Fig. 8 and Fig. 9 respectively. It is observed that the power obtained using W/T based encoder is approximately $2.5 \mathrm{~mW}$ which is comparatively much higher than that of the PSO based system. Fig. 10 and Fig. 11 depict the eye diagrams of both the system which proves that the performance of 2-D codes is better than 1-D codes. 


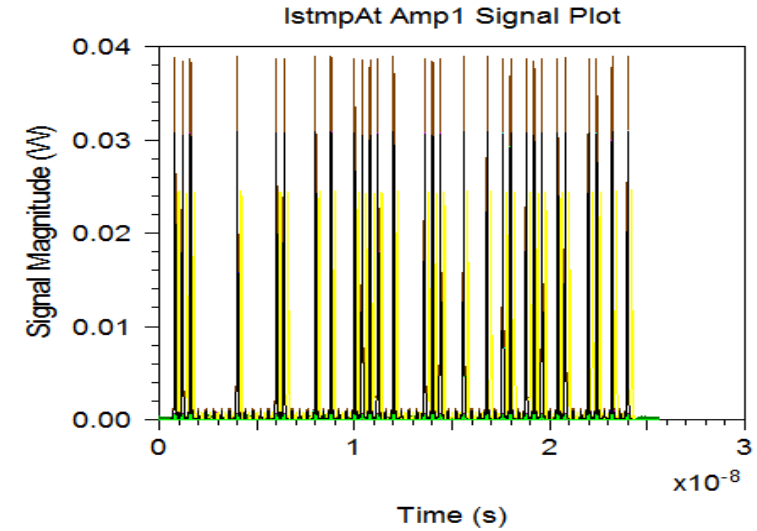

Fig. 7. Encoder output with W/T code.

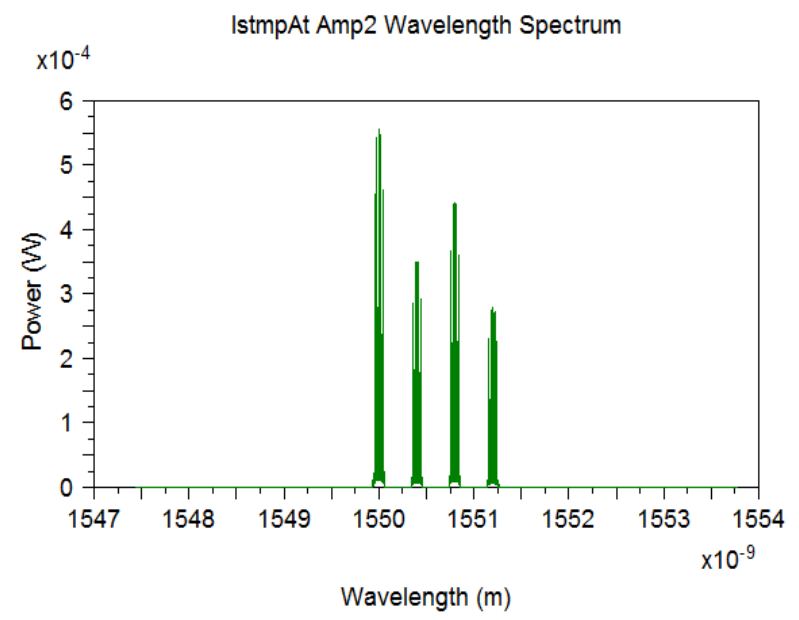

Fig. 8. Wavelength spectrum with PSO code.

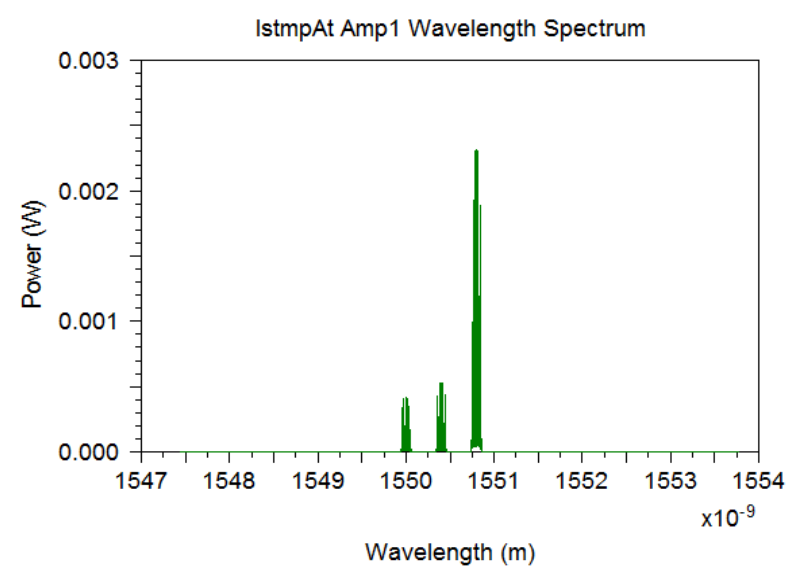

Fig. 9. Wavelength spectrum with W/T code.

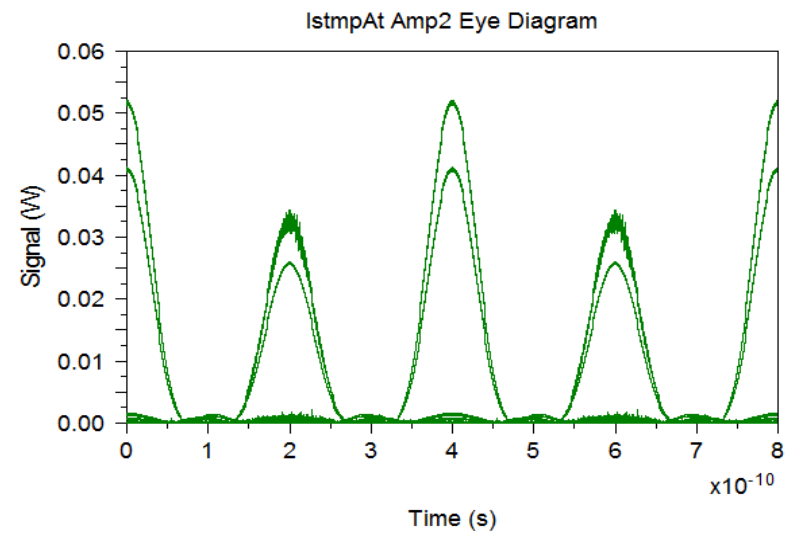

Fig. 10. Eye diagram with PSO code.

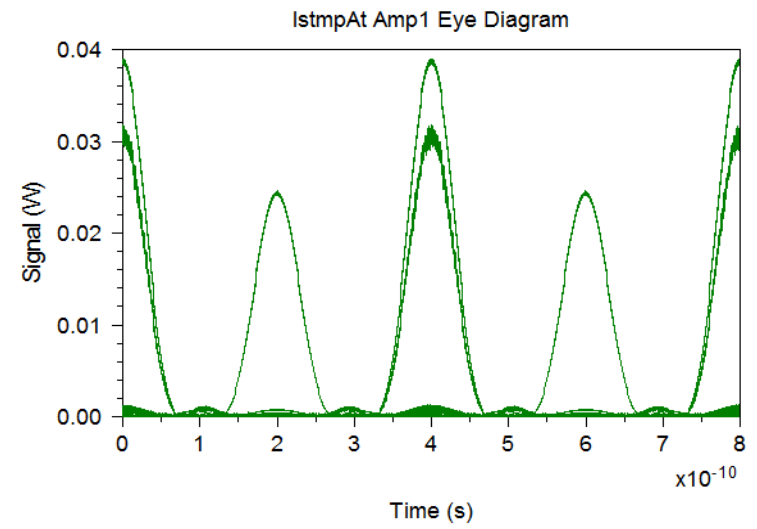

Fig. 11. Eye diagram with W/T code.

\section{CONCLUSION}

Two dimensional codes operate in both time and wavelength domain can be a good solution to the limitations offered by one dimensional codes. The Optical CDMA system has been designed using these W/T matrix code and WDM type components. The performance of W/T based encoder is analyzed and compared with PSO based encoder. It has been shown that two dimensional codes have better spectrum efficiency than one dimensional codes of the same cardinality. The performance of $\mathrm{W} / \mathrm{T}$ based encoder is comparatively better than that of one dimensional code.

\section{ACKNOWLEDGMENT}

Author Awab Fakih thanks Vidyalankar Institute of Technology for providing with the necessary software support.

\section{REFERENCES}

[1] P. R. Prucnal, M. A. Santoro, and T. R. Fan, "Spread spectrum fiberoptic local area network using optical processing," J. Lightw. Technol., vol. LT-4, no. 5, pp. 547-554, May 1986.

[2] T. Pfeiffer, B. Deppisch, M. Witte, and R. Heidemann, "Operational stability of a spectrally encoded optical CDMA system using inexpensive transmitters without spectral control," IEEE Photon. Technol. Lett., vol. 11, no. 7, pp. 916-918, Jul. 1999.

[3] T. Pfeiffer, J. Kissing, J.-P. Elbers, B. Deppisch, M. Witte, H. Schmuck, and E. Voges, "Coarse wdm/cdm/tdm concept for optical packet transmission in metropolitan and access networks supporting 400 channels at $2.5 \mathrm{~Gb} / \mathrm{s}$ peak rate," J. Lightw. Technol., vol. 18, no. 12, pp. 1928-1938, Dec. 2000.

[4] K. I. Kitayama, N. Wada, and H. Sotobayashi, "Architectural considerations for photonic IP router based upon optical code correlation," J. Lightw. Technol., vol. 18, no. 12, pp. 1834-1844, Dec. 2000.

[5] N. Wada, H. Harai, and F. Kubota, "40 Gb/s interface, optical code based photonic packet switch prototype," in Proc. Optical Fiber Commun. Conf., 2003, pp. 801-802.

[6] D. Gurkan, S. Kumar, A. Sahin, A. Willner, K. Parameswaran, M. Fejer, D. Starodubov, J. Bannister, P. Kamath, and J. Touch, "All-optical wavelength and time 2-D code converter for dynamically reconfigurable O-CDMA networks using a PPLN waveguide," in Proc. Optical Fiber Communication Conf., pp. 654-656.

[7] L. Tancevski, I. Andonovic, M. Tur, and J. Budin, "Hybrid wavelength hopping/time spreading code division multiple access systems," IEE Proceedings Optoelecton, vol. 143, no. 3, pp. 161-166, 1996.

[8] A.S. Holmes and R.R Syms, "All optical CDMA using 'quasi-prime' codes," IEEE/OSA Journal of Light Wave Technology, vol. 10, no. 2, pp. 279-286, Feb 1992.

[9] S. V. Maric, Z. I. Kostic, and E. I. Titlebaum, "A new family of optical code sequence for use in spread-spectrum fiber-optic local area 
network," IEEE Trans. on Communication, vol. 41, no. 8, pp. 1217-1221, Aug. 1993.

[10] G. C. Yang and W. C. Kwong, "Performance analysis of optical CDMA with prime codes," IEEE Electronic Letters, vol. 31, no. 7, pp. 569 570, Mar. 1995.

[11] W. C. Kwong, P. A. Perrier, and P. R. Pruncal, "Performance comparison of asynchronous and synchronous code division multiple-access technique for fibre-optic local area network," IEEE Trans. on Communication, vol. 39, no. 11, pp. 1625-1634, Nov. 1991

[12] A. J. Mendez, R. M. Gagliardi, V. J. Hernandez, C. V. Bennett, and W. J. Lennon, "Design and Performance Analysis of Wavelength/Time (W/T) Matrix Codes for Optical CDMA," IEEE Journal of Light Wave Technology, vol. 21, pp. 2524-2533, November 2003.

[13] V. J. Hernandez, A. J. Mendez, C. V. Bennett, R. M. Gagliardi, and W. J. Lennon, "Bit-Error-Rate Analysis of a 16-User Gigabit Ethernet Optical-CDMA (O-CDMA) Technology Demonstrator Using Wavelength/Time Codes," IEEE Photonics Technology Letters, vol. 17, no. 12, December 2005.

[14] H. S. Chung, S. H. Chang, B. K. Kim, and K. Kim, "Experimental Demonstration of Security-Improved OCDMA Scheme Based on Incoherent Broadband Light Source and Bipolar Coding," Optical Fibre Technology, vol. 14, pp. 130-133, 2008.

[15] T. Srinivas, K. Archana, P. Vijay, "Simulation and Performance analysis of OCDMA System base on 2-D W/T Codes," Annual IEEE, 2011.

[16] P. R. Prucnal, Optical Code Division Multiple Access: Fundamentals and Applications, CRC Press, 2006.

[17] J. S. Vardakas, "Performance Analysis of OCDMA PONs Supporting Multi-Rate Bursty Traffic," IEEE Transactions on Communications, vol. 61, issue 8, pp. 3374-3384, August 2013.

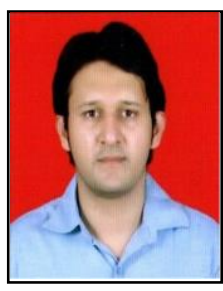

Awab Fakih is a member of IACSIT. He was born in Mumbai on $13^{\text {th }}$ November 1989. A. Fakih is currently pursuing his master degree in electronics and telecommunication at Vidyalankar Institute of Technology, Wadala, India. A. Fakih has completed his bachelor degree in engineering from Xaviers Institute of Engineering, Mahim, India in 2011.

$\mathrm{He}$ is currently working as an assistant professor at Anjuman-I-Islamic Kalsekar Technical Campus, Panvel, India. He has published 5 papers in international journals and 2 papers in international conferences. His interests lie in the field of optical fiber communication and antenna wave theory.

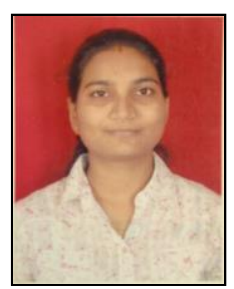

Shraddha Panbude has completed her M.Tech degree from B.A.T.U. Lonere in 2008. She has completed her B. Tech degree from B.A.T.U. Lonere in May 2002.

She is currently working as an assistant professor at Vidyalankar Institute of Technology, Wadala, India. She has a total of 9 years of teaching experience. She has published 2 papers in international journals and 3 papers in international conferences. Her interest lies in the field of optical communication.

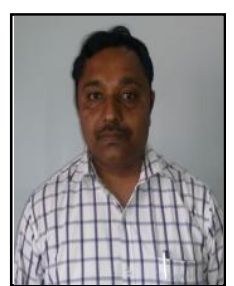

Santosh Jagtap has completed his M.Tech degree from VJTI in 2008. He completed his B.E. degree from KBP College of Engineering, Satara in 1998

He is currently working as an assistant professor at Vidyalankar Institute of Technology, Wadala, India $\mathrm{He}$ has a total of 14 years of teaching experience. He has published 5 papers in international journals and 3 papers in international conferences. His interests lie in the fields of Non linear fiber optics, RF communications and communication circuits. 